\title{
All-Optical Light Confinement in Dynamic Cavities in Cold Atoms
}

\author{
Jin-Hui Wu, ${ }^{1}$ M. Artoni, ${ }^{2,3}$ and G. C. La Rocca ${ }^{4}$ \\ ${ }^{1}$ College of Physics, Jilin University, Changchun 130023, People's Republic of China \\ ${ }^{2}$ Department of Physics and Chemistry of Materials \& CNR-INFM Sensor Laboratory, University of Brescia, Italy \\ ${ }^{3}$ European Laboratory for Nonlinear Spectroscopy, Firenze, Italy \\ ${ }^{4}$ Scuola Normale Superiore and CNISM, Pisa, Italy \\ (Received 17 January 2009; published 24 September 2009)
}

\begin{abstract}
We show how to realize in a cold atomic sample a dynamic magneto-optically controlled cavity in which a slow-light pulse can be confined and released on demand. The probe optical pulse is retrieved from the atomic spin coherence initially stored within the cavity and is subsequently confined there subject to a slow-light regime with little loss and diffusion for time intervals as long as a few hundred microseconds before being extracted from either side of the cavity. Our proof-of-principle scheme illustrates the underlying physics of this new mechanism for coherent light confinement and manipulation in cold atoms. This may ease the realization of nonlinear interactions between weak light pulses where strong atom-photon interactions are required for quantum information processing.
\end{abstract}

PACS numbers: 42.50.Gy, 42.70.Qs

Quantum networks relying on nodes linked by channels play an important role in the flourishing field of quantum computation and communications [1]. While channels transport qubits from site to site with high fidelity and distribute their entanglement across the entire network, nodes are used to generate, store, and manipulate quantum information. Photons are ideal and quite robust flying qubits for carrying quantum information between nodes, yet it is not easy to efficiently store and control them.

So far several interesting proposals have been put forward. One is to utilize the strong coupling of single photons and atoms in the setting of cavity quantum electrodynamics (CQED) [2]. Another one depends on the enhanced interaction between single photons and an atomic ensemble in the regime of electromagnetically induced transparency (EIT) [3]. In a common EIT scheme, the light signal carrying classical or quantum information is stored and subsequently retrieved from a spin coherence by adiabatically switching off and then on a travellingwave (TW) coupling beam [4-7]. It may be interpreted as the formation of a dark-state-polariton (DSP) whose photonic component is transferred into its atomic component and vice versa [8]. In this case the stored signal has no photonic component so that the usual nonlinear optical interactions cannot be implemented during storage. Another EIT scheme consists in retrieving a light signal from the spin coherence by switching on a perfect standing-wave (SW) grating beam with equal forward (FW) and backward (BW) components [9-11]. This gives rise to a stationary, rather than a propagating, light signal which suffers however loss while diffusing within the region where this SW grating is effective [12].

We propose here for the first time to use an ultracold atomic sample to realize a dynamically controlled cavity in which a propagating slow-light pulse can be confined and released on demand. The basic idea is to divide the ultra- cold atomic sample into three continuous regions by applying a local and well confined magnetic field. The central region, with a nonvanishing magnetic field, is the cavity within which the signal is slowed down and compressed through a TW coupling beam [13]. In the two contiguous regions where the magnetic field is absent, a SW grating creates instead two distributed Bragg mirrors which effectively prevent the signal from propagating $[14,15]$. Then, a light signal stored in atomic spin coherence may be retrieved and confined within the cavity by the two mirrors by a suitable time modulation of the magnetic field and the TW and SW beams. One main advantage of this scheme is that the signal has nonzero photonic components and suffers little loss and diffusion during confinement. After a desirable confinement time, we can release the light signal just by modulating the strength of one component of the SW grating, which partially or totally turns off the mirrors.

In order to implement such a scheme, we consider an ensemble of ultracold ${ }^{87} \mathrm{Rb}$ atoms (D1 line at $795 \mathrm{~nm}$ ) driven by three optical fields as in Fig. 1 where the three hyperfine levels $\left|5^{2} S_{1 / 2}, F=1\right\rangle, \quad\left|5^{2} S_{1 / 2}, F=2\right\rangle$ and $\left|5^{2} P_{1 / 2}, F=2\right\rangle$ and relevant magnetic sublevels $|0\rangle,|1\rangle$, $|2\rangle$ and $|3\rangle$ are also shown. All atoms are initially pumped into the sublevel $|0\rangle$ and kept at $\mu \mathrm{K}$ temperatures so that they can be assumed to be stationary to a very good approximation. Unlike in the mirrors region, the degeneracy of all sublevels is lifted within the cavity. In the presence of a magnetic field $B_{0}$, the frequency difference between adjacent sublevels is given by $\Delta_{1}=\mu_{B} g_{1} B_{0} / \hbar$ for the $\left|5^{2} S_{1 / 2}\right\rangle$ state and by $\Delta_{2}=\mu_{B} g_{2} B_{0} / \hbar$ for the $\left|5^{2} P_{1 / 2}\right\rangle$ state, with $g_{1} \cong 3 g_{2}$ [16]. Inside the cavity, the probe $\omega_{p}\left(\sigma^{+}\right.$polarized) resonantly couples the transition $|0\rangle \leftrightarrow|3\rangle$, the TW coupling $\omega_{c}$ ( $\pi$ polarized) resonantly couples the transition $|1\rangle \leftrightarrow|3\rangle$, while the SW grating $\omega_{d}$ ( $\sigma^{-}$polarized) is far detuned from the $|2\rangle \leftrightarrow|3\rangle$ transition. Inside the mirrors region, however, it is the $\mathrm{SW}$ grating $\omega_{d}$ 

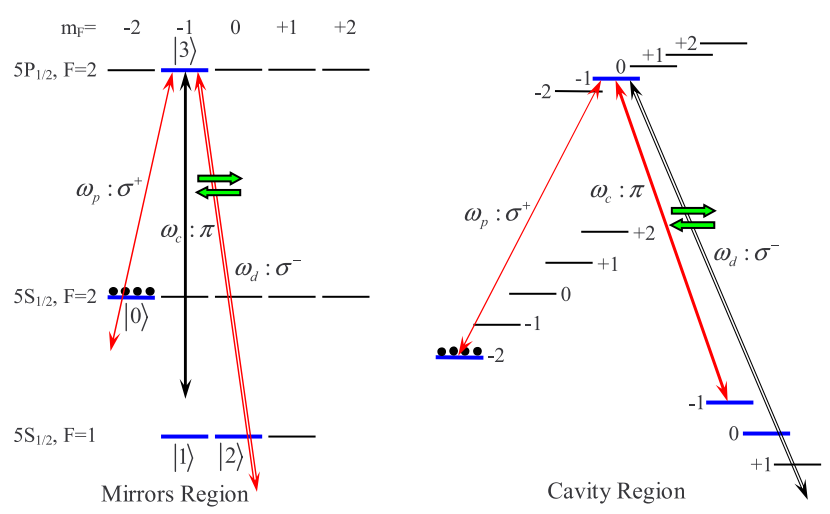

FIG. 1 (color online). Level scheme configuration for ${ }^{87} \mathrm{Rb}$ atoms in the presence (cavity region) and in the absence (mirrors region) of a static magnetic field. Circularly polarized probe and SW beams copropagate in the $+z$ direction parallel to the magnetic field, while the TW beam propagates in the $+x$ (or $+y$ ) direction and is linearly polarized parallel to the magnetic field. Thick blue lines denote the 4 magnetic sublevels coupled to the 3 optical fields, only two of which at a time (in red) are effective, respectively, in the mirrors or cavity region.

that effectively interacts with the probe $\omega_{p}$ at two-photon resonance, while the TW coupling $\omega_{c}$ is far detuned from its two-photon resonance with the probe $\omega_{p}$. In the cavity region the probe, coupling, and grating field detunings are taken, respectively, as $\Delta_{p}=\omega_{p}-\omega_{30} \cong 0, \Delta_{c}=\omega_{c}-$ $\omega_{31} \cong 0$, and $\Delta_{d}=\omega_{d}-\omega_{32} \cong 6 \Delta_{2}$. In the mirrors region, one then has $\Delta_{p}=\omega_{p}-\omega_{30} \cong 5 \Delta_{2}, \quad \Delta_{c}=$ $\omega_{c}-\omega_{31} \cong-4 \Delta_{2}$, and $\Delta_{d}=\omega_{d}-\omega_{32} \cong 5 \Delta_{2}$.

The propagation of a probe pulse through the cavity region - in the presence of the SW grating-and inside the mirrors region-in the presence of the TW coupling-is a fairly complicated problem to be computed with great accuracy. It turns out, however, that for a suitable range of the two-photon detunings $\Delta_{d}-\Delta_{p}$ and $\Delta_{c}-$ $\Delta_{p}$, the SW grating and the TW coupling may to a very good approximation be ignored, respectively, in the cavity and mirrors regions. This would enable one to reduce the original tripod dynamics [17] to that of a simpler lambda configuration [8], which will now be assessed.

The steady-state susceptibility for a lambda configuration composed of levels $|0\rangle,|1\rangle$, and $|3\rangle$ is

$$
\chi_{L 0}=\frac{N\left|d_{03}\right|^{2}}{\varepsilon_{0} \hbar} \frac{i \gamma_{10}^{\prime}}{\gamma_{10}^{\prime} \gamma_{30}^{\prime}+\Omega_{c}^{2}},
$$

where $N$ denotes the homogeneous atomic density, $\gamma_{10}^{\prime}=$ $\gamma_{10}+i \Delta_{c}-i \Delta_{p}$ and $\gamma_{30}^{\prime}=\gamma_{30}-i \Delta_{p}$ are complex dephasing rates while $\Omega_{c}=E_{c} d_{31} / 2 \hbar$ is the (real) Rabi frequency of the TW coupling. If we restrict ourselves for simplicity to the case of $\Delta_{c}=0$, the susceptibility for sufficiently small probe detunings and spin-coherence dephasings, i.e., $\left|\Delta_{p}\right| \ll \Omega_{c}$ and $\gamma_{10} \ll \gamma_{30}<\Omega_{c}$, can be written as

$$
\chi_{L 1} \simeq \frac{N\left|d_{03}\right|^{2}}{\varepsilon_{0} \hbar} \frac{\Delta_{p}+i\left(\gamma_{10}+\gamma_{30} \Delta_{p}^{2} / \Omega_{c}^{2}\right)}{\Omega_{c}^{2}} .
$$

The relevant steady-state susceptibility for the tripod configuration of Fig. 1, on the other hand, is given by

$$
\chi_{T 0}=\frac{N\left|d_{03}\right|^{2}}{\varepsilon_{0} \hbar} \frac{i \gamma_{10}^{\prime} \gamma_{20}^{\prime}}{\gamma_{10}^{\prime} \gamma_{20}^{\prime} \gamma_{30}^{\prime}+\gamma_{10}^{\prime} \Omega_{d}^{2}+\gamma_{20}^{\prime} \Omega_{c}^{2}},
$$

where the dephasing and Rabi frequency $\gamma_{20}^{\prime}$ and $\Omega_{d}$ are defined just as $\gamma_{10}^{\prime}$ and $\Omega_{c}$. Likewise, for sufficiently strong SW gratings and small detunings and dephasings, i.e., $\left|\Delta_{p}\right| \ll \Omega_{d}$ and $\gamma_{\{10,20\}} \ll \gamma_{30}<\Omega_{d}$, and provided that $\Omega_{c} \simeq \Omega_{d}<\Delta_{d}$ the susceptibility $\chi_{T 0}$ reduces to

$$
\chi_{T 1} \simeq \chi_{L 1}^{\prime}(1+\mathcal{E})+i \chi_{L 1}^{\prime \prime}(1+2 \mathcal{E}),
$$

where $\chi_{L 1}^{\prime}$ and $\chi_{L 1}^{\prime \prime}$ are, respectively, the real and imaginary parts of $\chi_{L 1}$. Under these conditions $\mathcal{E} \equiv\left(\Delta_{p} / \Delta_{d}\right) \times$ $\left(\Omega_{d}^{2} / \Omega_{c}^{2}\right) \cong \Delta_{p} / \Delta_{d}$ is rather small and it is clear that $\chi_{T 1}$ is to a very good approximation the same as $\chi_{L 1}$. The error, introduced by the far detuned SW grating, in the cavity transmissivity is $\delta T / T_{L 1}=2 \mathcal{E} k_{p} L \chi_{L 1}^{\prime \prime}$, which turns out to be negligible as the optical depth on EIT resonances is typically small for a cold atomic sample $\left(k_{p} L_{\chi_{L 1}^{\prime \prime}} \lesssim 0.1\right.$ for the parameters adopted in this letter). This means that for sufficiently small spin coherence dephasings and when $\left|\Delta_{p}\right| \ll \Omega_{c} \sim \Omega_{d}<\Delta_{d}$ one can safely neglect the SW grating and replace the tripod with a lambda configuration. More generally when $\Delta_{c} \neq 0$, the requirement for a negligible error in the cavity transmissivity becomes $\mid \Delta_{c}-$ $\Delta_{p} \mid \cong 0$ and $\left|\Delta_{d}-\Delta_{p}\right| \gg\left|\Delta_{c}-\Delta_{p}\right| \Omega_{d}^{2} / \Omega_{c}^{2}$. A similar conclusion is obtained when the mirrors probe reflectivity is instead examined [18]. In the mirrors region, one can neglect the TW coupling and again replace the tripod with a lambda configuration provided that $\left|\Delta_{d}-\Delta_{p}\right| \cong 0$ and $\left|\Delta_{c}-\Delta_{p}\right| \gg\left|\Delta_{d}-\Delta_{p}\right| \Omega_{c}^{2} / \Omega_{d}^{2}$.

In the mirrors region, the two nonvanishing spin and optical coherences can be expanded in the limit of a weak probe, respectively, as $\rho_{20}=\sum_{-\infty}^{+\infty} \rho_{20}^{(n)} e^{i 2 n k_{d} z}$ and $\rho_{30}=$ $\sum_{-\infty}^{+\infty} \rho_{30}^{(n)} e^{i(2 n+1) k_{d} z}$ [15]. The propagation of a probe pulse is described in terms of the spatial Fourier components $\rho^{n}$ through the following coupled Maxwell-Liouville equations

$$
\begin{aligned}
\partial_{t} \rho_{20}^{(n)}= & -\gamma_{20}^{\prime} \rho_{20}^{(n)}-i \Omega_{d-}^{*} \rho_{30}^{(n-1)}-i \Omega_{d+}^{*} \rho_{30}^{(n)}, \\
\partial_{t} \rho_{30}^{(n)}= & -\gamma_{30}^{\prime} \rho_{30}^{(n)}-i \Omega_{d+} \rho_{20}^{(n)}-i \Omega_{d-} \rho_{20}^{(n+1)} \\
& -i \Omega_{p+} \delta_{n, 0}-i \Omega_{p-} \delta_{n,-1}, \\
\partial_{z} \Omega_{p+}= & -\partial_{t} \Omega_{p+} / c+i \Delta k \Omega_{p+}+i \gamma_{30} \alpha \rho_{30}^{(0)} / 2, \\
\partial_{z} \Omega_{p-}= & +\partial_{t} \Omega_{p_{-}} / c-i \Delta k \Omega_{p-}-i \gamma_{30} \alpha \rho_{30}^{(-1)} / 2,
\end{aligned}
$$

where $\quad \alpha=\frac{N\left|d_{03}\right|^{2}}{\varepsilon_{0} \hbar} \frac{k_{p}}{\gamma_{30}}, \quad \Delta k=k_{p}-k_{d}$, and $\quad k_{p} \cong k_{d}$. Likewise, in the cavity region the probe propagation may 
be assessed only in terms of the TW coupling. In the same weak probe limit the relevant coupled Maxwell-Liouville equations are instead

$$
\begin{aligned}
& \partial_{t} \rho_{10 \pm}=-\gamma_{10}^{\prime} \rho_{10 \pm}-i \Omega_{c}^{*} \rho_{30 \pm}, \\
& \partial_{t} \rho_{30 \pm}=-\gamma_{30}^{\prime} \rho_{30 \pm}-i \Omega_{c} \rho_{10 \pm}-i \Omega_{p \pm}, \\
& \partial_{z} \Omega_{p \pm}=\mp \partial_{t} \Omega_{p \pm} / c \pm i \gamma_{30} \alpha \rho_{30 \pm} / 2,
\end{aligned}
$$

where $\Omega_{p^{+}}$and $\Omega_{p^{-}}$may simultaneously exist as a result of Bragg reflectance but remain uncoupled. By using Eqs. (5) truncated at a sufficiently large $|n|$ (e.g., $|n|=$ 30) and Eqs. (6), we can evaluate the probe propagation with boundary conditions at $z=0, z=L_{a}, Z=L_{b}$, and $z=L$, where $L_{a}$ and $L_{b}$ denote, respectively, the borderlines between the cavity and the mirrors region. The pulse dynamics is best illustrated (see Fig. 2) by separating the three sequential steps of storage, retrieval and confinement, and on-demand readout.

In the first step, we turn on the TW coupling to let the probe pulse propagate inside the atomic sample at an extremely slow velocity while being compressed there. The compression ratio is equal to the scaled probe group velocity $v_{g} / c$ and then depends on the Rabi frequency $\Omega_{c}$. After a given delay, we switch off the TW coupling to transfer the compressed probe pulse into the stationary spin coherence $\rho_{01}$ at the sample center.

In the second step, we create the cavity confined by two mirrors in the atomic sample by switching on first an accurately confined magnetic field, and then the TW and SW light beams. The compressed probe is retrieved by the TW coupling [19] and propagates at a very slow velocity $v_{g} \cong 139 \mathrm{~m} / \mathrm{s}$ in the same direction as before storage. As the retrieved probe hits one mirror, it is reflected so as to spatially oscillate back and forth within the cavity with negligible loss and diffusion. The oscillation frequency is inversely proportional to the probe group velocity and thus can be controlled by modulating the Rabi frequency $\Omega_{c}$.

In the third step, we illustrate a typical readout situation where we first reduce $\Omega_{d-}$ to $0.87 \gamma_{30}$ to partially reduce the mirrors reflectivity so that a half of the stored signal can be released. Then, we temporarily increase $\Omega_{d^{-}}$to $3.48 \gamma_{30}$ to recover the mirrors and generate another oscillation. Finally, we reduce $\Omega_{d-}$ to zero to release the other half signal in the same $+z$ direction. The total losses for the entire process shown in Fig. 2 are about $50 \%$ because the total energy of the two released wave packets is about $50 \%$ of the input energy. In an actual experiment, both optical and magnetic fields should be switched on and off adiabatically to avoid extra losses while great care should be devoted to the magnetic field stability and spatial confinement [20].

We finally examine in Fig. 3 the probe propagation dynamics within a steady cavity. The probe pulse is seen to undergo various oscillations with little loss and diffusion over an oscillation period $T_{\text {cav }}=2 L_{\text {cav }} / v_{g} \cong 10 T_{s}$. When
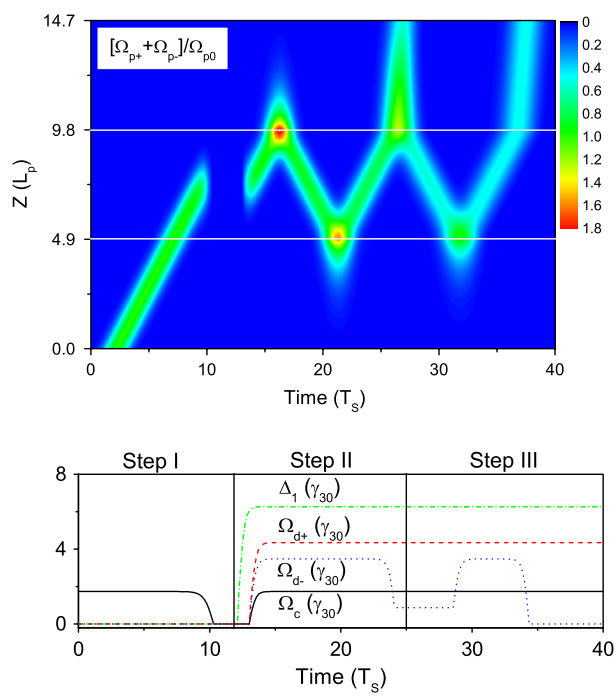

FIG. 2 (color online). Upper: An incident light pulse with a $1 / e$ half width of $T_{s}=12.65 \gamma_{30}^{-1}=2.20 \mu \mathrm{s}$ is all-optically confined in a cavity stretching from $4.9-9.8 L_{p}$ with $L_{p}=$ $v_{g} T_{s}=306 \mu \mathrm{m}$. The cavity is created by applying a magnetic field that Zeeman splits the relevant levels at the sample center while on-demand readout is achieved by modulating the backward (BW) component of the standing-wave (SW) grating. The probe pulse and the traveling-wave (TW) coupling are resonant $\left(\Delta_{p}=\Delta_{c}=0\right)$ in the cavity, but become off-resonant $\left(\Delta_{p}=\right.$ $\left.10.43, \Delta_{c}=-8.35\right)$ in the adjacent mirrors region. The SW grating detunings are instead $\Delta_{d}=12.62$ and $\Delta_{d}=10.53$ respectively in the cavity and mirrors regions. The sample consists of ultracold ${ }^{87} \mathrm{Rb}$ atoms with a resonant absorption length of $l_{a}=1 / \alpha=0.633 \mu \mathrm{m}=2.07 \times 10^{-3} L_{p} \quad$ and dephasings $\gamma_{10}=3.48 \times 10^{-5}, \gamma_{20}=3.48 \times 10^{-4}$. The probe, TW coupling, and SW grating wavelengths are, respectively, $\lambda_{p}=$ $794.983 \mathrm{~nm}, \lambda_{c}=794.969 \mathrm{~nm}$ and much the same value for $\lambda_{d}$ as the TW and SW light beams differ in frequency by $3 \Delta_{1}$. A small misalignment ( $0.06 \mathrm{rad}$ ) between the FW and BW components of the SW grating is employed to optimize the mirror reflectivity. Lower: Time modulation of the coupling and grating Rabi frequencies $\Omega_{c}$ (black) and $\Omega_{d \pm}$ (red and blue) as well as the Zeeman splitting $\Delta_{1}$ (green). All frequencies are in units of $\gamma_{30}=5.75 \mathrm{MHz}$

transmission $(91.0 \%)$ due to EIT in the cavity region and the mirrors Bragg reflectivity $(96.2 \%)$ are optimized as in Figs. 2 and 3, the intensity $\propto \Omega_{p \pm}^{2}$ decays to $10 \%$ of its initial value in about $90 T_{s}$ at an average decay rate of $\Gamma_{\text {cav }} \cong 0.002 \gamma_{30}$, becoming only 2.5 times larger when the spin coherence $\gamma_{10}$ is increased by an order of magnitude. Figure 3 displays roughly 4 reflections off the mirrors before the pulse intensity decreases by $1 / e$.

We have illustrated a scheme for on-demand readout of photonic excitations stored within a long-lived ground state spin coherence, where all-optically controlled readout both in direction and timing may easily be attained. Upon retrieval the excitation can be transferred to a cavity created right within the storing medium. Confinement takes 

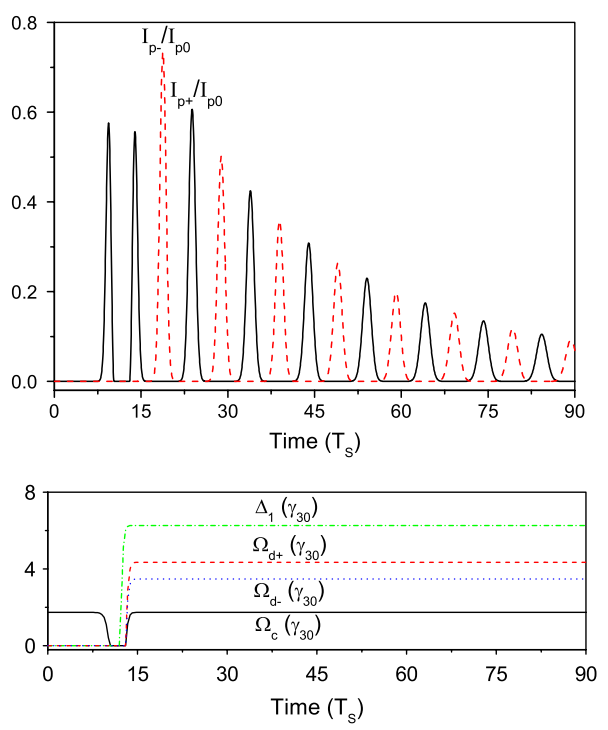

FIG. 3 (color online). Upper: Scaled intensity decay of the oscillating FW (black) and BW (red) probe components with the same parameters as in Fig. 2. Lower: Time modulation of the coupling and grating fields as well as the Zeeman splitting.

place in a well-defined slowly propagating spatial mode, at variance with the stationary mode observed, e.g., in [9], and suffers little loss over intervals of a small fraction of a millisecond. Such an efficient fairly long confinement, along with an easy on-demand readout of the stored field, could be used to appreciably enhance the optical nonlinear interaction between weak classical or quantum pulses and appears to be flexible enough for quantum information processing. Its extension to solid-state media, though may not be straightforward, would certainly be appealing. Nitrogen-vacancy centers in diamond, e.g., have intrinsically higher reflectivities and transmissivities [21] which would lead in fact to an overall enhancement of the confinement efficiency.

Jin-Hui Wu gratefully acknowledges the hospitality at Scuola Normale Superiore in Pisa and helpful discussions with G. Ferrari and M. Inguscio. This work is supported by NSFC-10874057, NCET-06-0309, DYSJ-20070121, NBRP-2006CB921103, the PRIN 2006-021037 Grant and the Azione Integrata IT09L244H5 of MIUR, the CNR contract 0008233 and the CRUI-British Council Partnership Program on "Atoms and nanostructures."

[1] H. J. Kimble, Nature (London) 453, 1023 (2008).

[2] A. D. Boozer, A. Boca, R. Miller, T. E. Northup, and H. J. Kimble, Phys. Rev. Lett. 98, 193601 (2007).
[3] M. Fleischhauer, A. Imamoglu, and J. P. Marangos, Rev. Mod. Phys. 77, 633 (2005).

[4] C. Liu, Z. Dutton, C. H. Behroozi, and L. V. Hau, Nature (London) 409, 490 (2001).

[5] T. Chaneliere, D. N. Matsukevich, S.D. Jenkins, S.-Y. Lan, T.A.B. Kennedy, and A. Kuzmich, Nature (London) 438, 833 (2005).

[6] J. Appel, E. Figueroa, D. Korystov, M. Lobino, and A. I. Lvovsky, Phys. Rev. Lett. 100, 093602 (2008).

[7] K.S. Choi, H. Deng, J. Laurat, and H. J. Kimble, Nature (London) 452, 67 (2008).

[8] M. Fleischhauer and M. D. Lukin, Phys. Rev. Lett. 84, 5094 (2000).

[9] M. Bajcsy, A.S. Zibrov, and M.D. Lukin, Nature (London) 426, 638 (2003).

[10] F.E. Zimmer, A. Andre, M.D. Lukin, and M. Fleischhauer, Opt. Commun. 264, 441 (2006).

[11] K. Hansen and K. Molmer, Phys. Rev. A 75, 053802 (2007).

[12] Our full calculation, i.e., Eqs. (5) with inclusion of higherorder spatial Fourier components, shows that the retrieved stationary signal exhibits severe loss and diffusion, at variance with a recent result [11] obtained under inaccurate approximations.

[13] L. V. Hau, S.E. Harris, Z. Dutton, and C.H. Behroozi, Nature (London) 397, 594 (1999).

[14] M. Artoni and G. C. La Rocca, Phys. Rev. Lett. 96, 073905 (2006).

[15] J.-H. Wu, M. Artoni, and G. C. La Rocca, J. Opt. Soc. Am. B 25, 1840 (2008).

[16] http://steck.us/alkalidata.

[17] E. Paspalakis and P. Knight, J. Mod. Opt. 49, 87 (2002).

[18] This is also supported by a numerical evaluation of the probe reflectivity and transmissivity carried out starting from Eqs. (1) and (3) through a Transfer-Matrix method. The mirrors reflectivity profile, e.g., obtained by taking $\Delta_{d} \simeq \Delta_{p} \simeq 2 \Omega_{c}$ and $\Delta_{p}-\Delta_{c} \simeq 3.6 \Omega_{c}$, turns out to be essentially the same as that obtained by ignoring the TW coupling beam in the mirrors region.

[19] The input and output signals have a frequency difference of $9 \Delta_{2}$ because of the application of the magnetic field prior to the retrieval.

[20] An estimate for the magnetic field fluctuations $\delta B_{0} / B_{0} \sim$ $\delta \Delta_{1} / \Delta_{1}$ may be obtained by taking a Zeeman splitting $\Delta_{1} / B_{0} \sim 0.7 \mathrm{MHz} / \mathrm{G}$ and an uncertainty $\delta \Delta_{1} \sim$ $0.07 \mathrm{kHz}$ which gives a contribution to $\gamma_{10}$ of $0.2 \mathrm{kHz}$. This yields a required field stability on the $0.1 \mathrm{mG}$ scale. In order to ease the stability and spatial confinement of the magnetic field, a configuration in which the central cavity and the two mirrors are built within three separated atomic samples may also be envisaged in a proof-of-principle experiment.

[21] J.-H. Wu, G. C. La Rocca, and M. Artoni, Phys. Rev. B 77, 113106 (2008). 